\title{
Multiples Myelom: Stammzelltransplantation lohnt sich auch bei Älteren
}

\section{Der Nutzen einer Transplantation hämatopoetischer Stammzellen (HSCT) ist bei jungen Patienten mit multiplem Myelom etabliert. Profi- tieren auch Ältere?}

D ie autologe HSCT ist die bevorzugte Therapie für jüngere Myelompatienten. Ihre Wirksamkeit bei älteren Patienten ist in klinischen Studien jedoch nicht ausreichend belegt. Aktuelle Untersuchungen haben allerdings gezeigt, dass das Verfahren auch bei älteren Patienten zunehmend häufiger zum Einsatz kommt. Daher wurde nun die Wirksamkeit bei älteren Patienten in einer Real-World-Studie untersucht.

Im SEER(Surveillance, Epidemiology, and End Results)-Register wurden 4.515 Patienten im Alter von $\geq 66$ Jahren identifiziert, bei denen zwischen 2000 und 2007 ein multiples Myelom diagnostiziert wurde und von denen $263(5,8 \%)$ eine HSCT erhalten hatten. Die mediane Be-

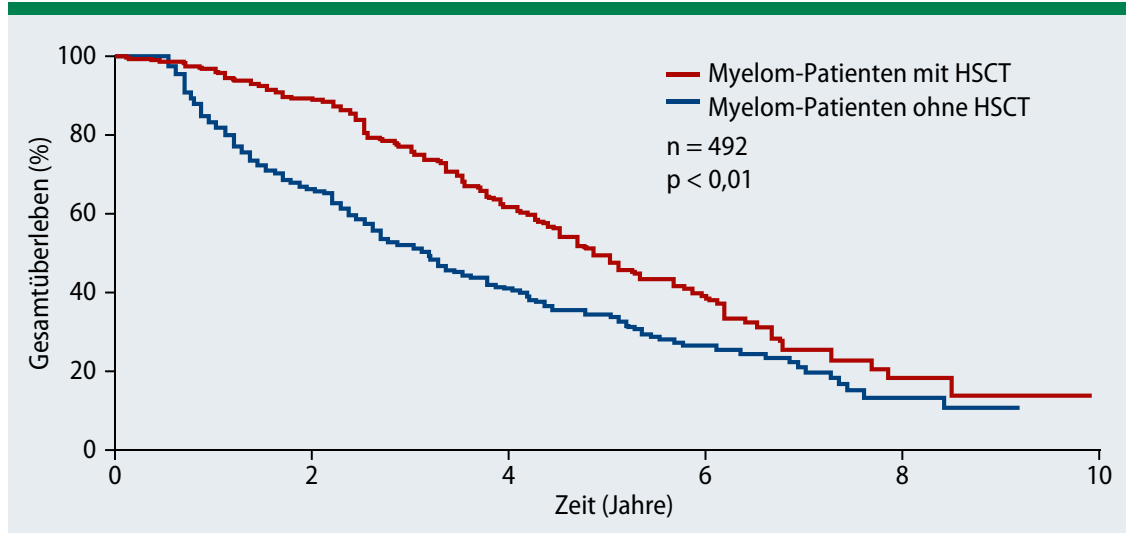

HSCT $=$ Transplantation hämatopoetischer Stammzellen

Abb. 1: Überleben der $\geq 66$ Jahre alten Patienten mit und ohne Stammzelltransplantation

\section{Zellmobilisation plus Chemotherapie: Kein Vorteil}

\section{Zur Mobilisierung autologer hämatopoetischer Stammzellen wird neben Wachstumsfaktoren heute oft auch eine Chemotherapie verabreicht. Hat dies auch Einfluss auf das Überleben von Myelompatienten?}

$\mathrm{D}$ urch eine Chemotherapie zusätzlich zu Wachstumsfaktoren (GF, ,growth factor") wie G-CSF („granulocyte-colony stimulating factor") oder GM-CSF (,granulocyte-macrophage colony-stimulating factor") können periphere Stammzellen schneller gewonnen und mehr CD34-positive Stammzellen refundiert werden als bei ausschließlicher Mobilisierung mit dem GF. In einer retrospektiven Analyse der Befunde von fast 1.000 Patienten gingen US-amerikanische Onkologen nun der Frage nach, ob es Unterschiede zwischen der Mobilisie-

rung durch GF mit und ohne Chemotherapie beim progressionsfreien (primärer Endpunkt) und Gesamtüberleben (sekundärer Endpunkt) gibt.

Ausgewertet wurden die Daten von insgesamt 968 Patienten mit multiplem Myelom, die an Zentren in den USA und Kanada innerhalb eines Jahres nach Diagnose eine Melphalan-Hochdosistherapie und eine autologe Stammzelltransplantation (ASCT) erhalten hatten. Zur Mobilisierung der Stammzellen wurde bei 519 Patienten nur ein Wachstumsfaktor (G-CSF oder GM-CSF) verwen- obachtungsdauer betrug 32 Monate. Patienten, die transplantiert wurden, waren mit höherer Wahrscheinlichkeit männlich, weiß, verheiratet, jünger und hatten weniger Komorbiditäten. Die Ergebnisse aller Analysen zeigten übereinstimmend, dass die Stammzelltransplantation das Überleben der Patienten signifikant verbesserte (Abb. 1). Die multivariate Analyse ergab eine Hazard Ratio [HR] von 0,582. Die mit anderen Methoden berechneten Hazard Ratios waren vergleichbar (Propensity-Score-Analyse: HR 0,572; Coarsened Exact Matching: HR 0,608 ; instrumentelle variable Analyse: HR 0,531; alle p-Werte $\leq 0,001$ ). Somit sind diese Überlebensdaten sehr aussagekräftig, da sie in allen statistischen Verfahren bestätigt wurden.

Fazit: Auch ältere Patienten mit multiplem Myelom profitieren von der Transplantation hämatopoetischer Stammzellen. Das Gesamtüberleben verbesserte sich dadurch signifikant.

Judith Neumaier

Winn AN et al. The Real World Effectiveness of Hematopoietic Transplant Among Elderly Individuals With Multiple Myeloma. J Natl Cancer Inst. 2015;107(8):djv139.

det, bei 449 Patienten wurde er mit Chemotherapie kombiniert, in $75 \%$ der Fälle mit Cyclophosphamid.

Nach der Transplantation erfolgte bei allen Patienten eine immunmodulatorische oder Bortezomib-basierte Therapie. Bei keinem der beiden Studienendpunkte errechneten die Onkologen signifikante Unterschiede zwischen beiden Strategien.

Fazit: Welche Strategie man zur Stammzellmobilisierung beim multiplen Myelom wählt, bleibt sich gleich. Das Ergebnis der ASCT ist auf lange Sicht nicht unterschiedlich.

Peter Leiner

Uy GL et al. Contribution of chemotherapy mobilization to disease control in multiple myeloma treated with autologous hematopoietic cell transplantation. Bone Marrow Transplant. 2015 Aug 24. [Epub ahead of print]. 